\title{
VNS Therapy
}

Can. J. Neurol. Sci. 2008; 35: 276-277

Since the July 1997 review of the American Academy of Neurology Therapeutics and Technology Subcommittee, vagus nerve stimulation (VNS) has been considered promising. ${ }^{1} \mathrm{~A}$ subsequent reassessment by the same Subcommittee in September 1999 concluded that seizure control with VNS was "comparable to that of new AEDs", while the gold standard remained temporal lobectomy in appropriate surgical candidates. $^{2}$ Since then little has changed, at least in North America, where VNS is thought to be as efficacious as, but more invasive than, the option of a newer AED, and this despite the claims of a cumulative effect in time with VNS. ${ }^{3}$ For the most part, use of this device in Canada has been limited to the most refractory epilepsy cases, often after surgical resection has failed.

However seizure frequency is just one variable to be considered in management of epileptic patients. Outcomes for any therapy should include health related quality of life (QOL) changes, reflected by improvement in social, cognitive and emotional well being, where problems are more common in the epileptic population. ${ }^{4,5}$ Anecdotal and literature support for QOL improvement with VNS stimulation exist, but the results of clinical trials do not strongly endorse these claims which are consistently independent of improved seizure control.5-7 Cognitive benefits are linked to enhanced memory consolidation and retention, and possibly to behavioural (autonomic and limbic) activation through VNS induced stimulation of the locus coeruleus. ${ }^{8,9}$ The issue of whether VNS has advantages beyond seizure control is accordingly important because it would justify its more invasive application to benefit QOL concerns when seizure control was not probable.

In this issue of The Canadian Journal of Neurological Sciences, McGlone et $\mathrm{al}^{10}$ set out to evaluate, in a novel manner, the effects of VNS therapy on QOL in a prospective case control paradigm, comparing results in the VNS complex partial seizure group with those of medically managed (MC) and cerebral surgical resection (RS) groups using the Quality of Life in Epilepsy-89 questionnaire, Geriatric Depression Scale, Wechsler Memory Scale-111, and the Memory Observation questionnaire, before VNS implantation and surgical resection and 12 months later, and twice in the medically treated group one year apart. The results included a reliable change index data analysis which provides an objective standard for interpretation of the magnitude of change on an individual case basis, and as well they preformed the usual between group analysis. Their conclusion that VNS: "adjunctive therapy for seizures did not change QOL, depressive affect, or objective memory scores over one-year more so than medical management alone" seems disappointingly negative. However, a number of qualifying comments need to be made to place these results in proper clinical perspective.

One of the apparent strengths of this study is that comparative groups of epileptic patients were used to help demonstrate a therapeutic effect. However the groups were not well matched apart from age and type of seizure. Scrutiny, especially of the VNS group clearly identifies a more severely compromised population, with five illiterate patients vs one MC, and two RS patients. Furthermore, seven VNS patients had not improved with prior surgical resection or callosotomy. These differences are important given the small numbers of patients in each arm of the study. With this level of cognitive dysfunction in the VNS cohort caregiver observations become more important, but are unlikely to refect accurately QOL changes. Moreover, in a patient population this severely affected by seizures, simple partial relief of seizures may not be clearly reflected in subjective QOL scores, or subjective and objective memory scores.

On a positive note, the VNS patients did no worse statistically on QOL and Memory scores than the surgical group (minor non significant changes in favour Working Memory post-op RS group, and Immediate Memory VNS and MC groups). This is somewhat encouraging given resective surgical therapy is considered to be the "gold "standard in treatment of medical refractory epilepsy. Also, when active controls (RS and MC) are used, differences between these groups and the study group are more difficult to demonstrate especially with uncontrolled medication changes.

Have we helped clarified the role of VNS therapy with this study? I think to some extent. It appears that VNS adjunctive therapy is just as safe as other medical and surgical options in terms of cognitive and behavioural side effects. We know that in the case of severe refractive epilepsy it will have a slight effect on seizure frequency $(17.7 \%$ in this study, $50-60 \%$ in other studies $^{3,11}$ ) which may grow in time. It may improve QOL in some individuals and in epileptic patients in general but it is not clear that these benefits are better than other active treatments. On an individual basis the prevention of status epilepticus with subsequent admission to hospital would be significant, while slight reduction in seizure frequency is unlikely to have an impact on QOL. This study included patients with partial seizures only, while the role of VNS in refractory idiopathic and symptomatic generalized epilepsy needs to be defined, ${ }^{11}$ and it may have a role in less severe focal epilepsy where medication side effects are problematic. Whatever adjunctive use VNS finds, its accepted application will depend on tolerable side effects. Although these were not addressed in this study, they are significant and may further impact negatively on QOL in the epileptic population. At this moment in time VNS therapy remains expensive, with limited availability, and modest effect. Clearly we need to cast our gaze elsewhere in search of more reliable and tolerable technological therapies. 


\section{REFERENCES}

1. Fisher RS, Krauss GL, Ramsay E, Laxer K, Gates J. Assessment: vagus nerve stimulation for epilepsy. Neurology. 1997;49:293-7.

2. Fisher RS, Handforth A. Reassessment: vagus nerve stimulation for epilepsy: a report of the Therapeutics and Technology Assessment Subcommittee of the American Academy of Neurology. Neurology. 1999;53:666-9.

3. Ardesch JJ, Buschman HP, Wagener-Schimmel LJ, van der Aa HE, Hageman G. Vagus nerve stimulation for medically refractory epilepsy; a long-term follow-up study. Seizure. 2007;16:579-85.

4. Wiebe S, Matijevic S, Eliasziw M, Derry PA. J Neurol Neurosurg Psychiatry. 2002;73:116-20.

5. McLachlan RS, Sadler M, Pillay N, Guberman A, Jones M, Wiebe $S$, et al. Quality of life after vagus nerve stimulation for intractable epilepsy: Is seizure control the only contributing factor? Eur Neurol. 2003;50:16-19.

6. Morrow JI, Bingham E, Craig JJ, Gray WJ. Vagal nerve stimulation in patients with refractory epilepsy. Effect on seizure frequency, severity and quality of life. Seizure. 2000;9:442-5.
7. Joyce A, Cramer BS. Exploration of changes in health-related quality of life after 3 months of vagus nerve stimulation. Epilepsy \& Behavior. 2001;2:460-5.

8. Ghacibeh GA, Shenker JI, Shenal B, Uthman B, Heilman KM. The influence of vagus nerve dtimulation on memory. Cogn Behav Neurol. 2006;19:119-22.

9. Henry TR, Bakay RAE, Votaw JR, Pennell PB, Epstein CM, Faber $\mathrm{TL}$, et al. Brain blood flow alterations induced by therapeutic vagus nerve stimulation in partial epilepsy: 1 . Acute effects at high and low levels of stimulation. Epilepsia. 1998;39:983-90.

10. McGlone J, Valdivia I, Penner M, Williams J, Sadler RM, Clarke DB. Quality of life and memory after vagus nerve stimulator implantation for epilepsy. Can J Neurol Sci. 2008;35:287-96.

11. Kostov H, Larsson PG, Roste GK. Is vagus nerve stimulation a treatment option for patients with drug-resistant idiopathic generalized epilepsy? Acta Neurol Scand Suppl. 2007;187:55-8. 